\title{
Summary of the Underwater Information Understanding Methods
}

\author{
Yi Kejia ${ }^{1}$, Pan Yue ${ }^{1}$, Zhang $\mathrm{Li}^{1,}$, ${ }^{\text {, Zhang Xiaoliang }}{ }^{1}$, Gao Kun ${ }^{1}$ \\ ${ }^{1}$ Science and Technology on Underwater Acoustic Antagonizing Laboratory, System engineering \\ research institute of CSSC, Beijing \\ azdsys_st@163.com
}

Keywords: Information Understanding, Data Mining, Knowledge Discovery, Information Fusion

\begin{abstract}
This paper meets the needs of underwater information warfare under development trends of knowledge centre war, starts from the concept of information understanding, combines with the characteristics of underwater information, deeply describes the underwater information understanding technology, lays the technical foundation for further research to the technical mechanism of underwater information understanding, improves the ability to identify underwater targets, judge and analyze the threat, get underwater awareness.
\end{abstract}

\section{Introduction}

Referring to the analysis of information understanding technology in "operational information" (the third volume of "2000-2035 US Navy technology"), information understanding technology uses the same basic theory of information extraction technology in business technology. Using automatic target recognition as the primary form can convert raw data to a higher level of information understanding, and it not only includes fixed feature extracted from sensor data, but also analyzes and ratiocinates the relationship and coexistence between the associated detections, eventually forms all-source automatic multi-sensor analysis and situational awareness.

Underwater information understanding technology is an important technical means to achieve underwater situational awareness, and the ultimate goal is to achieve real-time or previous all-source automatic multi-sensor analysis. For the particularity of underwater conditions, this paper analyzes the theories and methods of underwater information understanding technology. The main means to obtain underwater information is a variety of underwater acoustic transducers, such as warning detection sonar, submarine topography measurement equipment, hydrological environmental measurement equipment, navigation sensors, etc.

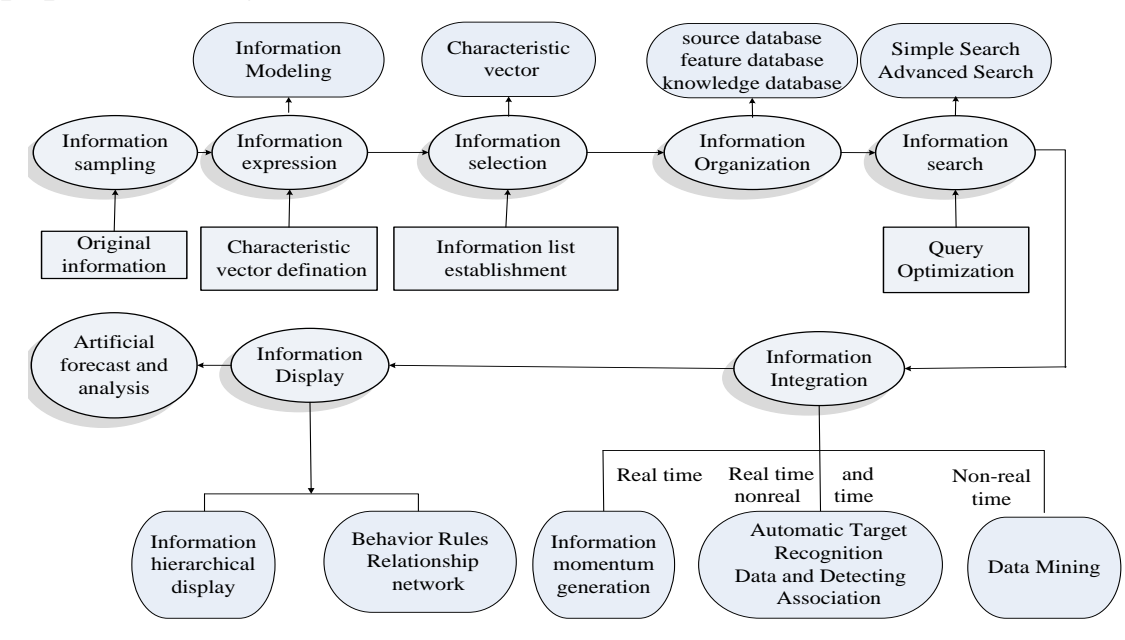

Fig.1 Information understanding system frame diagram

In the underwater battle, the process to get the sensor probe information, environmental information, intelligence information and other information can be understood as information 
sampling process. The raw information is stored in the information space and waits for further processing.

\section{Concept of Underwater Information Understanding ${ }^{[1]}$}

2.

Formation of cognitive ability may be represented by a pyramid-shaped structure, as shown in Fig.

The process (convert data obtained from the noise into information, upgrade it to knowledge, sublimate it to cognition), under the condition of huge amounts of underwater information, complex types, limited human attention source, needs an accelerator. The underwater information understanding technology is considered as an accelerator.

Knowledge is a higher level of information used to identify entities and their properties, is summarized and considered as correct factual information. Knowledge is the connection between the information. It must establish a connection between the information to form knowledge.Cognition refers to the process that people acquires and apply knowledge to know external things, or the process of information processing. Understanding is gradual understanding the relation of things. Understanding is to use existing knowledge and experience to acquire new knowledge and experience, incorporate the new knowledge and experience into existing knowledge and experience system. The combination of the old and new contacts forms a contact system.

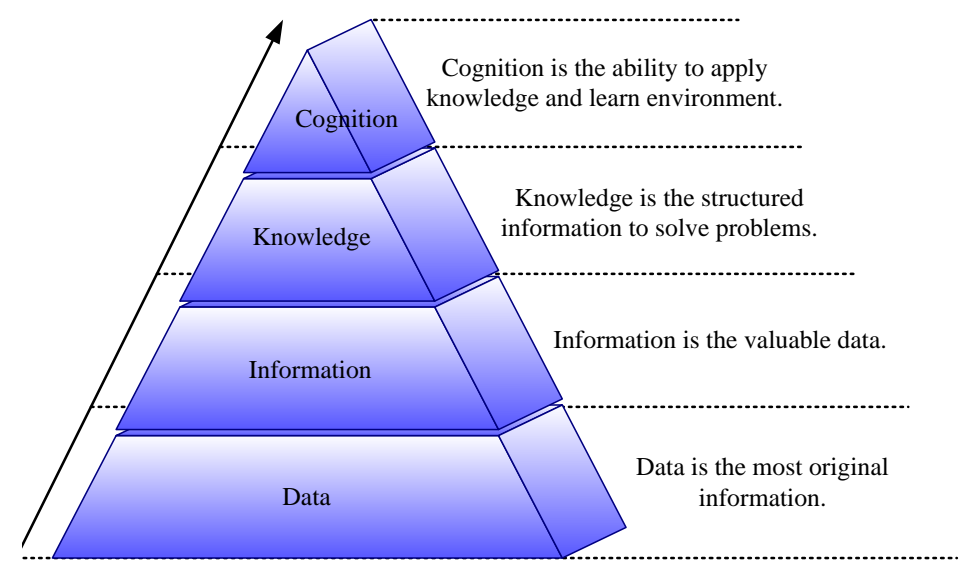

Fig.2 Pyramid-shaped structure of cognitive ability formation

Information understanding is a process to fuse data distributed in space and data distributed on time, and then form an interesting and situation associated image.Underwater information understanding technology is building the technology which assist human to cognize the information space of the underwater environment and target technology, is to tap the knowledge from underwater uncertain pieces of information, and the old and new contacts are organized into an interactive system technology which is conductive for staff to understand. The information processing and management toolset play the role to accelerate staff underwater situation awareness.

\section{Technology Involved in Underwater Information Understanding Method}

Underwater Information Expression Technology.Information expression is the process, based on certain rules and standards, that does a selection, a description or a record on information external characteristics and internal characteristics. Information expression technology in underwater information understanding, based on the metadata theory, on the basis of multi-source information modeling technology, obtains content characteristic information, semantic characteristic information and formatting characteristic information in the original multimedia data.

Underwater Information Selection Technology. Information selection technology mainly solves how to express data by using fewer dimensions. The reason is to make the subsequent analysis easier, improve the classification performance by a more stable representation and delete redundant 
or irrelevant information. In the literature about the multivariate analysis, methods for representing data by using reducing dimension is called ordination method or geometrical method, including principal component analysis and multidimensional scaling method. In the literature about the pattern recognition, it is called feature selection and feature extraction methods, including linear discriminant analysis and methods based on Karhunen-Loeve expansion.

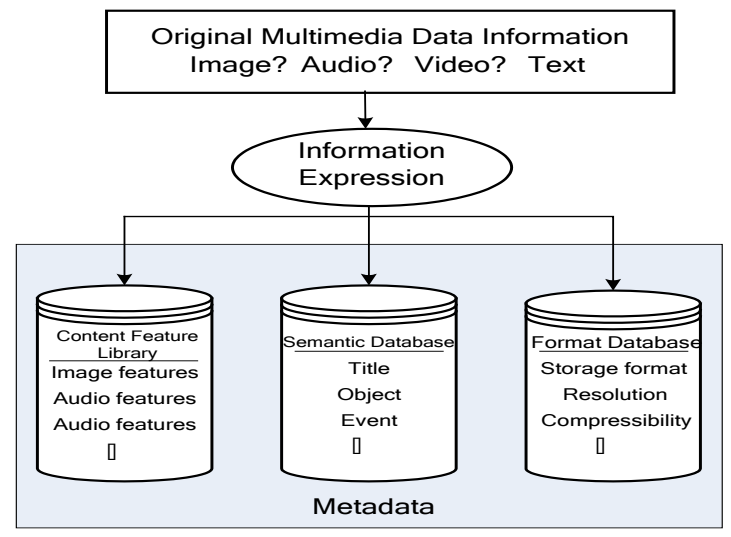

Fig.3 Underwater information expression process

Underwater Information Organization Technology. Underwater information organization technology is to store underwater information (different sources, different forms and different meanings) in database form. Information organization methods include single user database system, client-server database system, distributed database system, the $\mathrm{C} / \mathrm{S}$ structure database system and the $\mathrm{B} / \mathrm{S}$ structure database system. From the research view of generalized database, underwater information can also be regarded as a bigger, more complex database. Each access to information can be seen as a data source. And the structure is not the same for each data source, which constitutes a huge heterogeneous database environment.

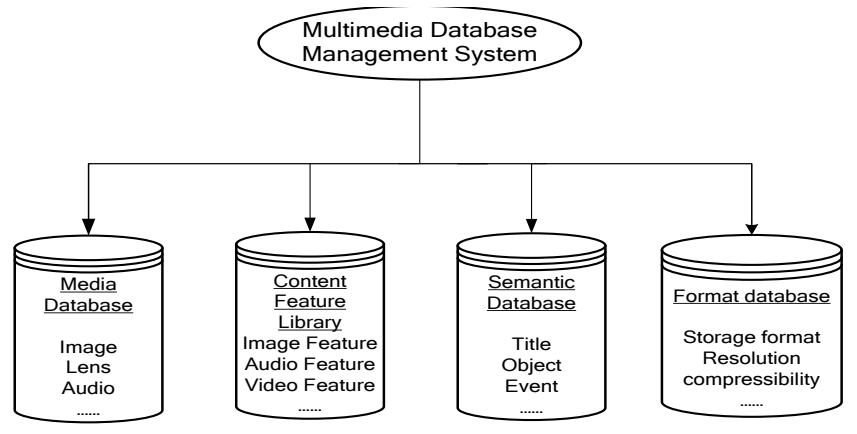

Fig.4 Multimedia database structure

Underwater Information Gathering Technology. The primary task is to establish a search engine which has the characteristics of meticulous and precision classification, low hardware requirements and subject-oriented. Using intelligence strategies such as artificial classification and feature extraction in the subject search can make the search engine more efficient and accurate.

Underwater Information Integration Technology. Information integration technology which belongs to underwater information understanding technology includes multi-source information fusion and knowledge discovery.Multi-source information fusion is an information processing method which expands for the system of the use of multiple it is also called a multi-source correlation, multi-source synthesis, sensor integrated or multi-sensor fusion, but the broader argument is far information fusion or multi-sensor information fusion, that is, information integration.

a. Correlation analysis

Correlation analysis is a mining technique which is applied in the data set to find an interesting data association. Usually association can be divided into simple association, temporal association, and causal association. APriori algorithm proposed by R.Agrawal is a well-known association rules 
discovery algorithm. Association rules discovery can be accomplished in two steps: first, identify frequent item of data sets by association rule mining, such as collection A and B satisfy the minimum support threshold, then produce association rules in the shape like $\mathrm{A}=>\mathrm{B}$. These rules also meet minimum confidence threshold which is predefined, and satisfies the probability of $B$ under the conditions of A.

\section{b. Classification}

Classification is to find a set of models or functions which can describe typical characteristics of the data set, in order to identify and classify the ownership or category of the unknown data, that is, to map the unknown instance to one of certain discrete categories. Classification model or function can be obtained by classification mining algorithms after learning from a group of its category attributing known training sample data. Classification model obtained by classification mining can be used to describe and output in a variety of forms.

\section{c. Cluster analysis}

Cluster analysis, first according to the basic cluster analysis principle that maximizes the similarity between the aggregate internal data objects, and minimizes the similarity between the aggregate objects. And using the formula of the similarity between the data object, the data object is divided into a plurality of data packets. Therefore, the similarity between data objects in a group is larger than the similarity between data objects in different groups. Each group acquired by cluster analysis can be regarded as a set of data objects belonging same category.

Underwater Information Display Technology. Information display provides data which has been processed to the operator, displays in the visual or multimedia mode, meanwhile establish the context data. According to the visualization techniques characteristics, applicability and other factors, type of visualization technology can be mainly divided into 6 categories, namely, standards ZD techniques, geometries techniques, icon-based techniques, graph-based techniques, hierarchical techniques and hybrid techniques. It is a fact that using a combination of multiple visualization technology in one or more windows can improve the visualization expression ability.

Artificial Performance Prediction. Artificial performance prediction is that the operator is considered as a component of information comprehension, and the operator can make a final decision on the system performance. In result interpretation and evaluation phases of the knowledge discovery process, users should be involved. Mode, discovered in data mining stage, through the users' assessment, may be redundant or irrelevant. Meanwhile, it's possible that mode does not meet user requirements, then it's necessary to make the entire discovery process return to the discovery phase.

General Foundation Technology. General foundation technology contains information network technology, data transmission technology and other common technology aiming to provide support for achieving information understanding.

\section{Conclusion}

Aiming to meets the needs of underwater information warfare under development trends of knowledge centre war, starts from the concept of information understanding, combines with the characteristics of underwater information, deeply describes the underwater information understanding technology, includes underwater information expression technology, underwater information selection technology, underwater information search technology, underwater information integration technology, underwater information display technology, artificial forecast technology and general foundation technology, lays the technical foundation for further research to the technical mechanism of underwater information understanding, improves the ability to identify underwater targets, judge and analyze the threat, get underwater awareness.

\section{References}

[1] Y. Pan: Concept and Method of Underwater Information Understanding. Ship Science and Technology Vol. 1 (2012), p. 82-84. 
[2] Z.G. Zhao, Z.H. Xiong and X.Y. Ke: Concept, Method and Application of Information Fusion. Beijing: National Defense Industry Press (2012).

[3] J.W. Han, K. Miceline: Concept and Technique of Data Mining. Beijing: Mechanical Industry Press (2001).

[4] X.Y. Zhou: Information Building Based on the Information Understanding. Beijing: China Renmin University Press (2005). 